\title{
Fattori Critici di Successo per il Laboratorio Clinico
}

\author{
Critical Success Factors for Clinical Laboratory
}

\author{
Piero Cappelletti ${ }^{1}$
}

Ricevuto: 15 giugno 2015 / Accettato: 19 giugno 2015

(C) Springer-Verlag Italia 2015

Riassunto I Fattori Critici di Successo (FCS) sono quelle poche caratteristiche che devono funzionare per consentire a un'organizzazione di raggiungere il successo. Essi sono necessari per determinare scelte strategiche adeguate agli obiettivi. Identificare i FCS del Laboratorio Clinico non è metodologicamente facile. Una ricerca narrativa indica in qualità e miglioramento continuo, innovazione e strategia $\mathrm{i}$ FCS della Medicina di Laboratorio.

Per "qualità" s'intende la "risposta al quesito clinico", che corrisponde alla soddisfazione delle necessità/richieste implicite/esplicite dei "clienti" della Medicina di Laboratorio, nella loro complessità. Il miglioramento continuo consente migliori esiti ed esperienze per il paziente attraverso cambiamenti sistemici di comportamenti e procedure della Medicina di Laboratorio, in particolare nella sua uscita dal "silo" in cui è spesso confinata.

Quando parliamo di "innovazione” in Medicina di Laboratorio intendiamo la sua capacità di disseminazione di strumenti tecnologici e diagnostici sempre più raffinati, ma anche la sua capacità di valutazione degli stessi e di creazione delle "evidenze" a sostegno delle novità tecnologiche e il trasferimento di queste conoscenze (knowledge management and translation) alla più ampia platea di pazienti, fornitori di servizio e stakeholders.

Per "strategia" in Medicina di Laboratorio intendiamo la capacità di pianificazione a lungo termine delle scelte strategiche e delle attività gestionali per il successo rispetto alla mission e alla vision, nel senso di "strategy-as-practice" dove l'innovazione organizzativa è il fattore critico. Essa, infatti, produce la separazione spazio-temporale del servizio al paziente, amplificando e complicando l'interfac-

$凶$ P. Cappelletti

pie.cappelletti@gmail.com

1 SIPMeL, Castelfranco Veneto, TV, Italia cia clinica-laboratorio con le problematiche della sicurezza, comunicazione e riorganizzazione intorno al paziente.

L'utilizzo dei FCS sopraricordati dovrebbe consentire ai Laboratori Clinici di diventare organizzazioni ad alta affidabilità e ad alto valore aggiunto.

Parole chiave Fattori critici di successo - Medicina di Laboratorio · Qualità · Innovazione · Strategia

Summary The Critical Success Factors (CSFs) are "those few things that must go well to ensure success" and allow strategic choosing. A non-systematic review identified as CSFs for Laboratory Medicine quality and continuous quality improvement, innovation, and strategy.

"Quality" is the answer to the clinical request that fits the implicit/explicit needs/requests of the complex "client" of the Laboratory Medicine, and "quality improvement" is the better outcomes and experience of the patient, achieved through systematic change of behavior and procedures, that overcome the barriers of the "silo" in which Laboratory Medicine is often confined.

"Innovation" is diffusion of new technologic and diagnostic interventions, and its validation, establishing evidences by structured methodologies as Evidence-based Laboratory Medicine. Moreover, knowledge management and translation to complex stakeholders are duties of Clinical Laboratory.

"Strategic planning and management" is not "something that organizations have", but "something that people do" in a vision of strategy-as-practice or "strategizing”, in which organizational innovation is the core of changing challenges. Converging technologies, i.e. miniaturization and information technology, determined the new context of relationship between patients and laboratory with the amplification of clinic-laboratory interface and consequential is- 
sues of safety, communication, and reorganization of service around the patient.

Using the above CSFs, the Clinical Laboratory should become a High-Reliability Organization and a High-value Organization.

Keywords Critical Success Factors · Laboratory

Medicine $\cdot$ Quality $\cdot$ Innovation $\cdot$ Strategizing

\section{Introduzione}

Il Laboratorio Clinico è definito dalla mission generale della Medicina di Laboratorio (fornire informazioni vitali per la salute degli esseri umani tramite un complesso di strumenti diagnostici fisici, chimici, biologici e molecolari) [1] e dalla vision specifica di ogni Laboratorio, dipendente dall'ambiente in cui opera quel singolo Laboratorio, che ne determinano le scelte strategiche competitive. Molte sono le teorie e le metodologie indicate per pianificare scelte strategiche, ma la maggior parte poggia sull'analisi delle competenze distintive o su processi di analisi collegiali e ragionate della realtà (tipo "SWOT analysis") per distillare/filtrare obiettivi smart e fattori critici di successo [2].

"Fattori Critici di Successo" (FCS; critical success factors, CSFs) è un termine gestionale utilizzato per identificare gli elementi necessari a un'organizzazione per centrare la propria mission. Secondo una prima definizione di RD Daniel nel 1960 si tratta di variabili chiave di numero limitato e di origine ambientale, che servono a selezionare le informazioni rilevanti nella creazione di sistemi informativi direzionali (inizialmente il termine fu utilizzato nell'ambito dell'Information Technology) di supporto alla pianificazione strategica. Nel 1979 JF Rockart, il guru dei FCS, riprese generalizzando la definizione: un numero limitato di aree il cui risultato soddisfacente assicura una performance competitiva all'organizzazione [3]. Per Brotherton e Shaw [4], FCS sono gli elementi essenziali che devono essere raggiunti affinché un'azienda o un suo settore produttivo sia in grado di esprimere il più competitivo ruolo di spinta produttiva. $\mathrm{Si}$ tratta di azioni e processi (non obiettivi), mobili (non statici), in quanto dipendenti dalla volontà aziendale e dall'ambiente vitale.

In queste definizioni è presente il concetto della differenza tra FCS e criteri di successo, legati piuttosto agli indicatori di performance (key performance indicators, KPI), che hanno a che fare con i risultati piuttosto che con le cause del successo imprenditoriale. Tuttavia è chiaro che vi è una stretta correlazione tra ciò che deve funzionare per ottenere il successo ("what must we do to be successful?", CSFs) e gli indicatori che il successo è stato raggiunto ("what indicates that we're winning?", KPIs) [5].

Queste definizioni, inoltre, si riferiscono a un concetto di competizione tipico dell'impresa privata e difficilmente trasferibile all'ambito dei servizi pubblici. Una più generale definizione di Boynlon e Zmud [6], "critical success factors are those few things that must go well to ensure success", è applicabile anche alle aziende pubbliche, la cui attività è caratterizzata da "intangibilità" (difficoltà di definire precisamente e di misurare il servizio), "eterogeneità" (dipendenza dall'interrelazione umana) e "inseparabilità" (la fornitura del servizio equivale ed è contestuale al suo consumo) [7], e ai servizi sanitari in particolare che sono, per di più, caratterizzati dalla diversità e numerosità degli stakeholders, dalla complessità delle azioni e dei "possessori" dei processi e dall'autonomia professionale di gran parte degli staff che in esso lavorano [8].

\section{Fattori Critici di Successo per il Laboratorio Clinico}

Identificare FSC per il Laboratorio Clinico non è facile. Ricerche nei database scientifici come PubMed dei termini "critical success factors" e "clinical laboratory" e/o "laboratory medicine" restituiscono circa 3000 risultati, quasi totalmente orientati a FSC di singoli contenuti o percorsi clinici o sperimentali; ricerche nel web con motori di ricerca come Google danno invece più di 4 milioni di risultati, per lo più inconsistenti rispetto al tema e quasi impossibili da verificare. $\mathrm{Ci}$ si deve affidare, quindi, a ricerche narrative e non sistematiche basate sulle conoscenze personali e sull'indagine critica delle referenze bibliografiche.

In un recente lavoro "Guidelines to set up a clinical laboratory", pubblicato sul blog di Clinicalabworld [9], viene definito un elenco di dodici FCS che rappresentano peraltro i temi generali che sintetizzano le attività di laboratorio: la tecnologia, la logistica riferita soprattutto al prelievo di sangue, la qualità dello staff principalmente sotto il profilo formativo, le caratteristiche del servizio analitico, il marketing, le norme e i vincoli pubblici, le minacce all'integrità professionale, il segmento di clientela servito, i costi, il posizionamento del Laboratorio nel sistema sanitario, il sistema informativo e il sistema qualità. Come si vede si tiene conto sia di fattori interni sia di fattori esterni al Laboratorio. Il lavoro cita uno statement dell'IFCC [10] che identifica i FCS per un Laboratorio da impiantare nei Paesi in via di sviluppo, ma che è utilizzabile in generale. In esso si ritrovano tre elementi comuni a molte segnalazioni di letteratura che si focalizzano su singoli FCS (la tecnologia con particolare enfasi per l'automazione, lo staff come competenze ed educazione, la logistica generale), ma anche due aspetti nuovi come l'accessibilità dei pazienti, segno di apertura del Laboratorio all'esterno del "silo" in cui è prevalentemente confinato, e la reputazione, che è un tipico elemento professionale e imprenditoriale.

Accenti in parte diversi sono presenti in un lavoro di una Società di consulenza privata svizzera [11]: accanto ad alcu- 
ne parole d'ordine in qualche modo oggi "dovute", come trasparenza e sostenibilità, il documento punta su un elemento assai concreto (Information and Comunication Technology) come strumento chiave del successo della tecnologia, centrando un dato assai ben conosciuto dalla pratica laboratoristica odierna [12], su un elemento già sottolineato quale lo staff e il suo coinvolgimento, e infine sulla tensione e sulla scelta dello strumento di miglioramento continuo, quale "specifico" del sistema qualità.

Infine, ricordo i risultati di un'analisi Metaplan [13] effettuata dalla SIMeL nel 2003, dalla quale emergevano come FCS: qualità analitica e clinica; capacità organizzative e gestionali; risorse umane (competenze ed educazione); $\mathrm{EB}(\mathrm{L}) \mathrm{M}$ e appropriatezza; innovazione diagnostica.

Filtrati e interpretati, dunque, i FCS per il Laboratorio Clinico possono essere sintetizzati in "qualità", "innovazione" e "strategia".

\section{Qualità e miglioramento continuo della qualità}

Quando parliamo di "qualità" in Medicina di Laboratorio facciamo riferimento alla definizione dell'Institute of Medicine (IOM), che la indica nel "grado di aumento delle probabilità di esiti di salute desiderati legati ai servizi sanitari per il singolo paziente e per la popolazione e in linea con la conoscenza professionale corrente" con le caratteristiche di sicurezza, efficacia clinica, centralità del paziente, tempestività, efficienza ed equità [14]. Queste caratteristiche identificano la "risposta al quesito clinico" e corrispondono alla soddisfazione delle necessità/richieste implicite/esplicite dei "clienti", nella loro complessità di pazienti, medici, personale di assistenza e stakeholders [15], sotto il profilo non solo analitico, non solo del processo totale (total testing process), non solo del prodotto, non solo dell'outcome, ma del servizio complessivo e complesso erogato in ogni situazione sia richiesto [15]. Sotto questo profilo, ben insisteva AB Burlina sul concetto "olistico" della qualità [16].

Il movimento "qualità" nelle organizzazioni produttive e dei servizi è stato descritto come un movimento pendolare tra ovest/est ed est/ovest, tra USA e Giappone, tra filosofia aristotelica-newtoniana e filosofia tao, che è iniziato con la tradizione ormai centenaria degli sforzi di miglioramento nell'industria manifatturiera americana, si è evoluto lungo $\mathrm{i}$ concetti di "Quality Control", "Quality Assurance", "Quality Management" e "Total Quality Management" per approdare alla teoria e pratica del "business excellence" [17]. La Qualità Totale (TQM) è caratterizzata: da una cultura aziendale basata sulla misurazione dei processi e degli esiti che produce qualità in quanto è un atteggiamento creativo, prospettico, proattivo focalizzato ai processi e alle interrelazio$\mathrm{ni}$, all'empowerment delle persone e alla produzione di elementi intangibili e invisibili cioè una cultura; dal focus sulla soddisfazione del cliente interno ed esterno, in un ambien- te costituito da un mondo in rapido cambiamento e con risorse decrescenti; da un sistema che si evolve imparando (learning organization) con elementi essenziali la teoria e la pratica del miglioramento continuo (continuous quality improvement) [18].

Il miglioramento continuo della qualità (QI) è un movimento, nato in USA con guru principale in D Berwick, come applicazione di TQM alla sanità (e talora confuso come sinonimo) focalizzato a migliorare la performance di tutti i processi, clinici e non, per ottenere esiti migliori in termini di salute, ma che poi è diventato una "filosofia di management integrato" di un'organizzazione che impara continuamente, trainata e sostenuta da un' adeguata leadership, aperta a ibridazioni e contaminazioni [19].

In effetti, le metodologie per la qualità, come le applicazioni six sigma ("zero defect") e lean thinking ("no waste") recentemente proposte per la sanità e viste talora come contrapposte a TQM sono invece da intendersi piuttosto come integrative, un insieme di concetti e strumenti utili a raggiungere il "business excellence", ma che hanno bisogno del framework di TQM come "integrated management philosophy", del suo focus sulla cultura organizzativa aziendale e sulle risorse umane [20, 21].

Il QI può essere definito come "migliori esiti ed esperienza per i pazienti conseguenti al cambiamento del comportamento e organizzazione del fornitore dei servizi sanitari attraverso l'uso di strategie e metodi di cambiamento sistematico" [22].

Nel settore pubblico, il "continuous improvement" dipende dalle peculiari caratteristiche del servizio (intangibilità, eterogeneità e inseparabilità) e deve tenere conto dei vincoli derivanti da obiettivi generali, impostazione politica, disponibilità di risorse. Tuttavia l'analisi di letteratura [7] è stata in grado di individuare pochi e significativi FCS validi anche per il settore pubblico: l'impegno al QI del top management; una chiara mission e un commitment alla soddisfazione del cliente di tutta l'organizzazione; l'uso dello strumento della pianificazione strategica e la cultura del cambiamento.

Un recente lavoro della RAND [23] ha individuato tre caratteristiche essenziali per riconoscere un QI: sistematico uso dei dati per guidare le attività, disegno del miglioramento tenendo conto della situazione specifica, utilizzo di metodi iterativi di sviluppo e controllo come il PDSA. Inoltre, altre caratteristiche con buon potere identificativo sono: focalizzarsi al cambiamento della routine, avere un set di specifici cambiamenti e un numero di specifici obiettivi predefiniti, fornire feedback agli implementatori e utilizzare team multidisciplinari. Infine conta produrre dati su che cosa produce il cambiamento e descrivere il contesto. In questo elenco non compare la "cultura" del miglioramento perché gli esperti della RAND sostengono che essa è essenziale per il successo e il mantenimento di QI, ma non è essenziale per la sua definizione. Ruth Boaden e colleghi, nello stilare il 
report "Quality improvement: theory and practice in healthcare" [21] indicano le caratteristiche del QI: occuparsi dei processi clinici e organizzativi contestualmente; privilegiare il processo di miglioramento piuttosto che il singolo metodo o approccio; è essenziale la forza della committenza e fattore critico è l'abilità della leadership.

Un tema centrale è se il QI sia efficace nella pratica per migliorare le capacità professionali e gli esiti di salute. La Cochrane Collaboration ha recentemente rilanciato un Protocollo [24] con questi obiettivi, la cui conclusione non è ancora stata presentata, alla luce delle evidenze limitate in numero, non quantitative, spesso non peer-reviewed, prevalentemente amministrative, molte volte non traslate in programmi effettivi per i pazienti, non durevoli nel tempo. Tuttavia, ancora nel 1998 Blumenthal e Kilo [19] sono stati in grado di elencare come successi di QI molti progetti individuali, l'accettazione del metodo da JCAHO, l'adozione di un linguaggio comune (e quindi una cultura comune), il superamento del concetto limitato di "quality assurance" e di "error blaming" per rivolgersi alla qualità totale e alla ricerca delle cause profonde dell'errore, il diffondersi delle " $p a$ tient survey" per valutare la soddisfazione del cliente. E nel 2005 Shojania e Grimshaw [25] hanno potuto riassumere i punti di effectiveness del QI: utilizzare interventi multifattoriali, consci che non esistono bacchette magiche; puntare sul cambiamento dei comportamenti dei fornitori del servizio; agire a livello di "disease management" con coordinamento di un team in collaborazione con il fornitore prossimale del servizio sanitario (es. il medico di medicina generale).

\section{Innovazione}

Quando parliamo di "innovazione" in Medicina di Laboratorio intendiamo la sua capacità di disseminazione di strumenti tecnologici e diagnostici sempre più raffinati, ma anche la sua capacità di valutazione degli stessi e di creazione delle "evidenze" a sostegno delle novità tecnologiche [26].

Secondo Schumpeter [27] "innovazione" è un'invenzione più il suo sfruttamento commerciale, una nuova idea ma trasferita nel contesto economico, dandole un contenuto pratico, utile e redditizio. Molti punti della teoria generale dell'innovazione interessano da vicino la Medicina di Laboratorio: la classificazione delle innovazioni tecnologiche (di prodotto o servizio e di processo) e non tecnologiche (organizzative e di marketing) [17]; i modelli "technology push" (nuovi biomarcatori e test) [26], ma anche "demand pull" (necessità diagnostiche emergenti; nuove forme organizzative per il servizio al paziente) [28], talora misti (disponibilità tecnologica più richiesta sanitaria come nel caso del POCT) [17]; la tipologia incrementale (il sincretismo tecnologico degli analizzatori ematologici) [29] o radicale (cambio di mercato come quello seguito all'uso di ICT in medicina o alla miniaturizzazione tecnologica) [30].
Schumpeter è fondamentale per la teoria dell'innovazione anche per un secondo concetto fondante: l'importanza della tecnologia ("il fatto tecnico") come fattore di cambiamento nel sistema economico a breve e fattore di crescita, di progresso nel lungo periodo [27]. Secondo CP Price le principali esperienze di innovazione in Laboratorio sono riferite all'automazione, all'introduzione dei POCT (miniaturizzazione), all'introduzione di nuovi marcatori e, prospetticamente, alle nanotecnologie. Sono le innovazioni "distruttive" - che rompono la struttura a silo della medicina di laboratorio-le più promettenti [30].

Price e St John [31] hanno recentemente definito i criteri che permettono di riconoscere una vera innovazione (cambiamento negli outcomes) in Medicina di Laboratorio: un bisogno clinico prima non soddisfatto; un'utilità definita scientificamente e presente nei percorsi trasversali; evidenze generate in termini di outcome e di cost-effectiveness e trasferite (business case) nei percorsi con cambio dei processi e riallocazione delle risorse; un piano di implementazione condiviso con gli stakeholders e adozione secondo specifici obiettivi; un appropriato disinvestimento di servizi non più richiesti.

Secondo Omachonu ed Einspruch [32] l'innovazione in sanità è caratterizzata dall'introduzione di "un nuovo concetto, idea, servizio, processo o prodotto teso a migliorare trattamenti, diagnosi, educazione, risultati, prevenzione e ricerca con obiettivi di lungo periodo di miglioramento della qualità, sicurezza, esiti, efficacia e costo".

In Medicina l'emblema dell'innovazione è la Medicina Traslazionale (translation medicine, TM) [27]. Il flusso della medicina traslazionale inizia con l'individuazione di quale trattamento sia da considerare, continua con la valutazione della sua efficacia e del perché esso funzioni (validazione) e infine con l'applicazione, l'identificazione di come applicarlo all'intera popolazione, in un processo che è definito come complesso, cumulativo, che necessita di tempo ed è spesso imprevedibile nei risultati. La Medicina Traslazionale è passata da una semplice e generica definizione ("the conversion of scientific discovery into health improvement") alla complessità dei passaggi attraverso due blocchi (definiti T) successivi del "bench-to-bedside" (T1) e del "reserch-into-practice" (T2) fino alla consapevolezza di un continuиm marcato da tre o addirittura quattro aree e barriere: T1 o fase traslazionale dagli studi di base e pre-clinici all'applicazione clinica (studi preclinici e trials di fase 1 e 2); T2 espande le scoperte alla comunità dei pazienti (trials di fase 3 e 4 e studi osservazionali); T3 è la fase orientata alla diffusione e disseminazione nella pratica; $\mathrm{T} 4$ focalizzata a tramutare in politica sanitaria quanto scoperto e confermato nelle fasi precedenti [27].

I compiti traslazionali della Medicina di Laboratorio sono sostanzialmente due: (1) operare la diffusione e implementazione dell'innovazione (biomarcatori o strumenti) 
modulandone uso e significato; (2) collaborare a creare le evidenze nella fase dell'impatto con la realtà clinica dapprima selezionata e poi diffusa per definire linee guida e percorsi assistenziali [26, 27].

Nella creazione delle evidenze è necessario conoscere quali metodologie tra EBM (evidence-based medicine), CER (comparative effectiveness research) e HTA (health technology assessment) rispondono adeguatamente ai quesiti se l'innovazione possa funzionare (efficacy), se funzioni nella pratica (effectiveness) e se sia preferibile (value). Recentemente Luce et al [33] hanno proposto una valutazione ben argomentata. In sintesi tutte rispondono alla domanda di "effectiveness", ma a livello di "decision making" solo EBM; nessuna al tema "efficiency", se non indirettamente EBM tramite RCT (randomized clinical trial), SRE (systematic review of evidence) e linee guida; e per il tema del valore, sicuramente HTA a livello di generazione e sintesi di evidenze, con minore forza EBM a livello di decision making. Tuttavia molte questioni restano aperte, in particolare in riferimento ai costi: il rapporto tra HTA e linee guida; il rapporto tra HTA e EBM; le caratteristiche degli studi CER. Per la Medicina di Laboratorio la metodologia migliore, più conosciuta e più utilizzata è la $\mathrm{EB}(\mathrm{L}) \mathrm{M}$. Sono però necessari criteri e metodi che la rendano ben fondata: per esempio, in un recentissimo lavoro dello "European Group on Tumor Markers" [34], sono elencati gli standard e le linee guida da seguire per ogni passaggio della validazione dei nuovi marcatori oncologici.

Infine, la traslazione in medicina (la diffusione dell'innovazione) ha a che fare, in particolare nella fase $\mathrm{T} 2$ "campusto-clinic", con la traslazione di conoscenza (generazione, condivisione e applicazione di conoscenza) [35]. La Knowledge Translation (KT) di successo in T1 dipende da fattori "supply" o "push" (scoperte e invenzioni industriali) e da fattori "demand" o "pull" (bisogni di salute), ma in fase T2 dipende da fattori "push" (evidenze disponibili, messaggi appropriati, opinion leader) e "pull" (rappresentanti locali, supporto politico, presenza strategica nelle aree decisionali) [27]. Si tratta di fattori critici che vanno presidiati, che la Medicina di Laboratorio ben conosce e in cui può avere un ruolo centrale [36]. Infatti, le relazioni tra Medicina di Laboratorio e stakeholders nell'innovazione sono complesse, coinvolgendo i ricercatori e le aziende costruttrici, i venditori, il paziente, i fornitori di servizi sanitari e in primis i medici, gli amministratori aziendali e regionali del servizio sanitario, i referenti politici e sociali del servizio sanitario. Infine, va sottolineato come l'innovazione in Medicina di Laboratorio non sia mai esclusivamente l'introduzione di un nuovo test, in relazione alla selezione, fornitura e interpretazione dello stesso, senza tenere conto degli aspetti organizzativi che ne consentono l'outcome, uscendo dal "silo" che ne impedisce il contatto diretto con i bisogni del paziente [31].

\section{Strategia}

Quando parliamo di "strategia" in Medicina di Laboratorio intendiamo la capacità di pianificazione a lungo termine delle scelte strategiche e delle attività gestionali per il successo rispetto alla mission e alla vision. Le "converging technologies" di Burtis [37] e in particolare i fenomeni della miniaturizzazione e dello sviluppo di "information and comunication technology" (ICT), innovazioni radicali secondo Clayton Christenson [30], hanno determinato la rottura spazio-temporale del rapporto medico-paziente e, per la Medicina di Laboratorio [36], l'amplificazione geometrica dell'interfaccia clinica-laboratorio con il portato inevitabile dei problemi a garantire la qualità/sicurezza ovunque nella rete, della moltiplicazione e complessità delle abilità comunicative e dell'impegno a costruire un'organizzazione sanitaria veramente disegnata sulle necessità del paziente cliniche (traslazionalità) e di qualità vita (trasformazione dell'organizzazione). In Medicina e in Medicina di Laboratorio l'innovazione tecnologica è il fattore determinante dei cambiamenti organizzativi e di servizio al paziente [17]. Il progressivo aumento della complessità delle innovazioni tecnologiche si sostanzia in successive generazioni di automazione, che a loro volta consentono/determinano modalità organizzative diverse del Laboratorio. Descritta in questo modo, l'evoluzione organizzativa del Laboratorio Clinico appare dominata dalla tecnologia in un purissimo modello "technology push". Tuttavia, dagli anni Ottanta in poi la tempestività dei risultati, il servizio per l'utente e il concetto di una sanità non per silos ma costruita intorno al paziente sono i drivers potenti del cambiamento del Laboratorio, associando un modello "demand pull" a quello "technology driven" [17].

Per fronteggiare questo radicale cambiamento è necessaria una strategia generale per la Medicina di Laboratorio e specifica per ciascun Laboratorio, cioè un piano d'azione di lungo termine utilizzato per impostare e successivamente coordinare le azioni tese a raggiungere lo scopo predeterminato, una "different activity in different way", come dice Michel Porter [2]. Secondo l'evoluzione del concetto, come proposto da Paula Jarzabkowski [38], lo "strategizing" (strategy-in practice) è qualcosa che le organizzazioni sono, non che le organizzazioni hanno, ne è cioè un fattore costitutivo, potenziando il filone di pensiero sulla centralità delle risorse umane e portando alla consapevolezza di dover fondere la "conoscenza" quale principale asset strategico delle organizzazioni con lo "strategizing" inteso come un processo di apprendimento continuo. Lo "strategizing", in questo senso, sta all'intersezione della strategia, delle pratiche e degli attori delle stesse, come un loro prodotto di sintesi. Si tratta di concetti che sono da un lato l'evoluzione dei concetti di organizzazione innovativa e dall'altro il completamento della linea culturale del miglioramento della quali- 
tà [17]. L'innovazione tecnologica è il core dello strategizing del Laboratorio [36].

Le prospettive del futuro cambiamento organizzativo dell'ospedale, indicate da Lega nel rapporto OASI del 2011 [39], valgono anche per la Medicina di Laboratorio, dove il cambiamento ha già interessato asset management, knowledge management e disease management [17].

Il cambiamento di "asset management" (modulazione della gestione operativa) si è esplicato nelle reingegnerizzazioni operative "technolgy-driven" (centralizzazioni/decentralizzazioni), ma anche nelle riorganizzazioni logistiche dipartimentali del core business e delle specialità con aree unitarie per l'erogazione dei servizi ambulatoriali e dell'urgenza e l'unitarietà delle risorse strategiche, in primis il LIS ma anche Sistema Qualità e Formazione [17]. Ciò che manca è la costruzione delle reti di attività non solo tra Laboratori ma prettamente cliniche (cardiologiche, oncologiche ecc.), in cui il Laboratorio declinato nelle modalità più "effective" abbia un suo ruolo riconosciuto.

Il cambiamento del "disease management" in Laboratorio si è sviluppato con la "traslazione" di marcatori e procedure "evidence-based" quale stimolo per l'innovazione del sistema ospedaliero e, sotto il profilo organizzativo, con la riorganizzazione a matrice delle attività dove al flusso operativo dettato da affidabilità, sicurezza e tempestività del dato, garantito da organizzazioni compatte e lean e governato dalle nuove professionalità manageriali si intersecano perpendicolarmente i saperi e le competenze specialistiche per l'introduzione di nuovi test, la validazione patient-oriented, la verifica collaborativa con il clinico della rispondenza dei risultati e degli esiti specialistici alle necessità del paziente singolo [36]. Ciò che va completato è un grande lavoro all'interfaccia clinico-laboratoristica con la verifica continua dell'efficacia e appropriatezza delle prestazioni, in particolare attraverso l'uso non episodico del confronto interno al team (il grande tema della comunicazione) e delle verifiche esterne (feedback e audit con medici, infermieri e pazienti) e con ripensamenti continui dell'operatività rivolta al paziente.

Il "knowledge management" (sviluppo, gestione e riorganizzazione delle competenze, delle specializzazioni e dei saperi) è stato ed è un punto centrale delle riorganizzazioni che non sono state solamente delle re-ingegnerizzazioni, ma una vera trasformazione dei saperi, competenze e comportamenti (per il cambiamento del focus di attività dei dirigenti e per l'emergere di una nuova figura di TSLB) [17] in una cultura comune [36] per realizzare, laddove leader e team hanno funzionato, equipe multidisciplinari e multiprofessionali che si esprimono in attività condivise in modo collaborativo secondo linee dettate dalla mission e dalla responsabilità clinico-organizzativa [1].

Dunque vi è in atto, anche in Medicina di Laboratorio, la trasformazione del concetto di organizzazione da una visione tradizionale tayloristica o fordista, condensata dalla metafora dell'organizzazione-macchina, a una visione innovativa, rappresentata dalla metafora della organizzazionecultura. Competenze e ruoli si ridefiniscono continuamente costruendo un vero team, dove peraltro il leader mantiene un ruolo centrale, "trasformazionale", nell'indicare la rotta (mission, vision, strategia), nell'innovazione e nello sviluppo delle competenze [36]. La qualità totale, nella sua accezione più vasta, è sorretta anche da una Total Quality Leadership [40], che si esplica sia nel "process management" (proattivo, coinvolgente il team, basato su misure oggettive, continuo) sia nello "strategic planning" (il processo che guida a una vision del futuro e ne sviluppa i passi per raggiungerlo) e "strategic management" (articola i legami tra la strategia e le decisioni gestionali giornaliere). Il leader, "coach and counselor", incoraggia ognuno e ne sviluppa le competenze e crea un ambiente pronto alla partecipazione e all'innovazione [17, 36].

\section{Conclusioni}

In un mondo dominato dalla complessità e dall'accelerazione del cambiamento [41], i FCS sopradescritti dovrebbero consentire di costruire Laboratori che raggiungano le caratteristiche delle organizzazioni altamente affidabili (High-Reliability Organizations, HRO) [42] e delle organizzazioni ad alto valore aggiunto (High-Value Healthcare Organizations, HVHO) [43].

Weick e Sutcliffe [44] hanno definito le caratteristiche e i comportamenti che caratterizzano le HRO. Gli autori descrivono un ambiente di "collective mindfulness" in cui i cinque principi dell' "alta attendibilità" sono: avere sempre in mente la qualità e la sicurezza; non semplificare procedure e comportamenti, e riconoscerne il valore per consentire identificazioni precoci dei rischi; essere capaci di riconoscere con grande sensibilità minacce e allarmi anche lievi e inapparenti in piccoli cambiamenti; essere resilienti; riconoscere le competenze ("deference to expertise, defence by expertise"), non necessariamente attraverso la tradizionale gerarchia organizzativa ma utilizzando chi sa. Chassin e Loeb [42] hanno recentemente identificato, attraverso una ricerca sistematica, le condizioni per essere HRO: il commitment della leadership all'obiettivo di zero rischio per il paziente, in cui sono coinvolti alta direzione, top e middle management in particolare i medici; la strategia della qualità e del miglioramento continuo e l'IT quale supporto imprescindibile; una vera cultura diffusa e interiorizzata della sicurezza, composta di fiducia, accountability, identificazione e rafforzamento delle sue caratteristiche critiche e valutazione iterativa; utilizzo di metodi e strumenti efficaci, come l'impiego combinato di tre strumenti di miglioramento (lean, six sigma, change management), definito "robust process improvement". 
D'altra parte, Bohmer et al [43] hanno identificato le quattro abitudini che appaiono essere sempre contemporaneamente presenti nelle organizzazioni sanitarie di alto valore (HVHO): pianificazione per problemi clinici con specificazione di percorsi e mappe personalizzate per singoli pazienti o gruppi per patologia; costruzione di "microsistemi" per rispondere alla personalizzazione degli interventi e armonizzazione degli stessi attraverso budget, processi, educazione e struttura del team; misurazione di processi ed esiti per controllo interno e gestione delle performance e integrazione con le alte priorità imprenditoriali e politiche per una totale "accountability" verso gli stakeholders; un sapere clinico non meramente individuale ma condiviso dall'organizzazione, continuamente messo in discussione, innovato e migliorato.

\section{Conflitti di interesse Nessuno.}

\section{Bibliografia}

1. Cappelletti P (2010) Medicina di Laboratorio. In: Galzigna L, Plebani M (eds) Trattato Italiano di Medicina di Laboratorio. Biochimica clinica generale, vol I. Piccin Nuova Libraria, Padova

2. Porter ME (1996) What is strategy? Harv Bus Rev 74:61-78

3. Rockart JF (1979) Chief executives define their own data needs. Harv Bus Rev 57:81-93

4. Brotherton B, Shaw J (1996) Towards an identification and classification of critical success factors in UK hotels Plc. International Journal of Hospitality Management 15:113-135

5. http://www.productiveflourishing.com/the-difference-betweencritical-success-factors-and-key-performance-indicators/\#sthash. 7DQPa2Dg.dpuf (Accesso 3 giugno 2015)

6. Boynton AC, Zmud RW (1984) An assessment of critical success factors. Sloan Management Review 25:17-27

7. Fryer KJ, Antony J, Douglas A (2007) Critical success factors of continuous improvement in the public sector. A literature review and some key findings. The TQM Magazine 19:497-517

8. The Health Foundation (2013) Quality improvement made simple. http://www.health.org.uk/publications/quality-improvementmade-simple/ (Accesso 3 giugno 2015)

9. Guidelines to set up a clinical laboratory. https://www.facebook. com/clinlabworld.blogspot.in/posts/1065862023441627 (Accesso 3 giugno 2015)

10. de Kievet W, Frank E, Stekel H (2008) Essential of clinical laboratory management in developing countries. http://www.ifcc. org/media/185572/2008\%20-\%20C-CLM\%20Monograph.pdf (Accesso 3 giugno 2015)

11. Schmidt M, Asaert W, Early J (2015) Laboratory excellence. Proven approaches to achieving and sustaining operational excellence in the laboratory environment. http://www.lodestonemc.com/files/ pdf/white\%20papers/WP-Laboratory\%20Excellence-120703.pdf (Accesso 3 giugno 2015)

12. Dorizzi RM (2007) Una roadmap per la information technology in Medicina di Laboratorio. RIMeL/IJLaM 3:75-81

13. Cappelletti P (2004) Metaplan ${ }^{\circledR}$ : un nuovo metodo di diagnosi e cura dei mali della Medicina di Laboratorio. Riv Med Lab-JLM $5: 249$

14. Institute of Medicine (2001) Crossing the quality chasm: a new health system for the 21 st century. The National Academies Press, Washington, DC
15. Cappelletti P (2005) La risposta della Medicina di Laboratorio al quesito clinico. RIMeL/IJLaM 1(Suppl.):15-23

16. Burlina AB (1993) La visione olistica della qualità. Med Lab 3:77-78

17. Morandini M (2014) Innovazione organizzativa in Medicina di Laboratorio. Riv Ital Med Lab 10:1-5

18. Hackman JR, Wageman R (1995) Total quality management: empirical, conceptual, and practical issues. Adm Sci Q 40:309-342

19. Blumenthal D, Kilo CM (1998) A report card on continuous quality improvement. Milbank Q 76:625-648

20. Andersson R, Eriksson H, Torstensson H (2006) Similarities and differences between TQM, six sigma and lean. The TQM Magazine 18:282-296

21. Boaden R, Harvey G, Moxham C et al (2008) Quality improvement: theory and practice in healthcare. NHS Institute for Innovation and Improvement/University of Manchester Business School, Coventry

22. Øvretveit J (2009) Does improving quality save money? A review of the evidence of which improvements to quality reduce costs to health service provides. The Health Foundation, London

23. Rubenstein L, Khodiakov D, Hempel S et al (2014) How can we recognize continuous quality improvement? Int J Qual Health Care 26:6-15

24. Brennan S, McKenzie JE, Whitty P et al (2013) Continuous quality improvement: effects on professional practice and healthcare outcomes (Protocol). The Cochrane Collaboration. Wiley, London

25. Shojania KG, Grimshaw JM (2005) Evidence-based quality improvement: the state of science. Health Aff (Millwood) 24:138-150

26. Cappelletti P (2009) La Medicina Personalizzata fra ricerca e pratica clinica: il ruolo della Medicina di Laboratorio. RIMeL/IJLaM 5(Suppl.):26-32

27. Cappelletti P (2013) Innovazione ed evidenze in Medicina di Laboratorio. Riv Ital Med Lab 9:177-184

28. Cappelletti P (2008) Come cambia la Medicina di Laboratorio. RIMeL/IJLaM 4(Suppl.):26-30

29. Cappelletti P (2006) Perché una storia dell'ematologia di laboratorio. RIMeL/IJLaM 2:196-198

30. Price CP (2010) Roots, development and future directions of laboratory medicine. Clin Chem Lab Med 48:903-909

31. Price CP, St John A (2014) Innovation in healthcare. The challenge for laboratory medicine. Clin Chim Acta 427:71-78

32. Omachonu VK, Einspruch NG (2010) Innovation in healthcare delivery systems: a conceptual framework. The Innovation Journal: The Public Sector Innovation Journal 15:1-20

33. Luce BR, Drummond MF, Jönsson B et al (2010) EBM, HTA, and CER: clearing the confusion. Milbank Q 88:256-276

34. Duffy MJ, Sturgeon CM, Sölétormos G et al (2015) Validation of new cancer biomarkers: a position statement from the European group on tumor markers. Clin Chem 61:809-820

35. Dorizzi RM (2005) La consulenza e la knowledge translation in Medicina di Laboratorio. RIMeL/IJLaM 1(Suppl.):60-71

36. Cappelletti P (2012) Scenari e ruoli della Medicina di Laboratorio al tempo della crisi: da Modena a Salerno ed oltre. Riv Ital Med Lab 8:183-189

37. Burtis CA (1996) Converging technologies and their impact on the clinical laboratory. Clin Chem 42:1735-1749

38. Jarzabkowski P, Spee AP (2009) Strategy-as-practice: a review and future directions for the field. International Journal of Management Reviews 11:69-95

39. Lega F (2012) Oltre i pregiudizi e le mode: natura e sostanza dell'innovazione organizzativa dell'ospedale. In: Cantù E (ed) L'aziendalizzazione della sanità in Italia. Rapporto OASI 2012. EGEA, Milano

40. Øvretveit J (2009) Leading improvement effectively. Review of research. The Health Foundation, London 
41. Cappelletti P (2014) La complessità e la Medicina di Laboratorio. Riv Ital Med Lab 10:187-192

42. Chassin MR, Loeb JM (2013) High-reliability health care: getting there from here. Milbank Q 91:459-490
43. Bohmer RM (2011) The four habits of high-value health care organizations. N Engl J Med 365:2045-2047

44. Weick KE, Sutcliffe KM (2007) Managing the unexpexcted. Jossey-Bass, San Francisco 